\title{
Erratum to: Decoding Prejudice Toward Hispanics: Group Cues and Public Reactions to Threatening Immigrant Behavior
}

\author{
Todd K. Hartman • Benjamin J. Newman • \\ C. Scott Bell
}

Published online: 11 August 2013

(C) Springer Science+Business Media New York 2013

\section{Erratum to: Polit Behav \\ DOI 10.1007/s11109-013-9231-7}

Unfortunately, some of the entries in Table 3 have been incorrectly listed under the headings in the original publication.

The corrected version of Table 3 is given below.

The online version of the original article can be found under doi:10.1007/s11109-013-9231-7.

T. K. Hartman ( $\square)$

Department of Government and Justice Studies, Appalachian State University, Boone, NC, USA

e-mail: hartmantk@appstate.edu

\section{B. J. Newman}

Department of Political Science, University of Connecticut, Stamford, CT, USA

e-mail: benj.newman@uconn.edu

C. S. Bell

Department of Political Science, Florida State University, Tallahassee, FL, USA

e-mail: scbell@fsu.edu 
Table 3 Mediated effects of experimental treatments on policy attitudes

\begin{tabular}{|c|c|c|c|c|c|c|}
\hline & \multirow{2}{*}{\multicolumn{2}{|c|}{$\frac{\text { Effect on mediator }}{\text { Violation seriousness }}$}} & \multicolumn{4}{|c|}{ Effect on policy attitudes } \\
\hline & & & \multicolumn{2}{|c|}{ Deport illegals } & \multicolumn{2}{|c|}{ Border fence } \\
\hline \multicolumn{7}{|l|}{ I. Overstay Visa Experiment } \\
\hline Group cue treatment & $.09 * *$ & $(.03)$ & .03 & $(.18)$ & -.18 & $(.14)$ \\
\hline Violation seriousness & & & $2.36 * * *$ & $(.35)$ & $2.32 * * *$ & $(.28)$ \\
\hline \multicolumn{7}{|c|}{ Mediated effects of group cue treatment } \\
\hline Total effect & & & .09 & & .02 & \\
\hline Indirect effect & & & $.07 * *$ & & $.07 *$ & \\
\hline Prop. total effect mediated & & & .87 & & 4.68 & \\
\hline \multicolumn{7}{|l|}{ II. Under the Table Experiment } \\
\hline Group cue treatment & $.06 *$ & $(.03)$ & .07 & $(.17)$ & -.12 & $(.14)$ \\
\hline Violation seriousness & & & $2.40 * * *$ & $(.44)$ & $2.25 * * *$ & $(.33)$ \\
\hline \multicolumn{7}{|c|}{ Mediated effects of group cue treatment } \\
\hline Total effect & & & .09 & & .01 & \\
\hline Indirect effect & & & $.06^{* *}$ & & $.05^{*}$ & \\
\hline Prop. total effect mediated & & & .69 & & .82 & \\
\hline \multicolumn{7}{|l|}{ III. Foreign Flag Experiment } \\
\hline Group cue treatment & $.22 * * *$ & $(.05)$ & -.22 & $(.17)$ & .05 & $(.14)$ \\
\hline Violation seriousness & & & $1.32 * * *$ & $(.22)$ & $1.05 * * *$ & $(.18)$ \\
\hline \multicolumn{7}{|c|}{ Mediated effects of group cue treatment } \\
\hline Total effect & & & .02 & & .10 & \\
\hline Indirect effect & & & $.10 * * *$ & & $.08 *$ & \\
\hline Prop. total effect mediated & & & 4.21 & & .78 & \\
\hline \multicolumn{7}{|l|}{ IV. Foreign Team Experiment } \\
\hline Group cue treatment & $.09 *$ & $(.04)$ & .01 & $(.16)$ & -.00 & $(.13)$ \\
\hline Violation seriousness & & & $.84 * *$ & $(.28)$ & $1.09 * * *$ & $(.24)$ \\
\hline \multicolumn{7}{|c|}{ Mediated effects of group cue treatment } \\
\hline Total effect & & & .03 & & .04 & \\
\hline Indirect effect & & & .03 & & $.04^{\dagger}$ & \\
\hline Prop. total effect mediated & & & .93 & & 1.00 & \\
\hline
\end{tabular}

$\mathrm{N} \sim 275$. Entries are the direct and indirect effects from 8 path models estimated using Imai et al.'s (2011) mediation package in R. The direct effects for (1) the binary treatments on the continuous mediators are OLS coefficients, and (2) the mediators on the categorical outcome variables are probit or ordered probit coefficients. The indirect effects are the change in the probability of supporting a restrictive immigration policy corresponding to a change in the value of the perceived offence mediator produced by moving from the control to treatment conditions.

${ }^{\dagger} p<.10, * p<.05$, ** $p<.01$, *** $p<.001$ level. Significance levels are based upon two-tailed hypothesis tests 